\title{
Hydrophilic Ionic Liquids as Ingredients of Gel-Based Dermal Formulations
}

\author{
Dorota Dobler, ${ }^{1,4}$ Thomas Schmidts, ${ }^{1}$ Christina Zinecker, ${ }^{1}$ Peggy Schlupp, ${ }^{1}$ Jens Schäfer, ${ }^{3}$ and Frank Runkel ${ }^{1,2,4}$
}

Received 11 March 2015; accepted 24 September 2015; published online 1 October 2015

\begin{abstract}
Ionic liquids (ILs) have several properties that offer many advantages in dermal drug delivery systems. Depending on the chemical structure, ILs can be used for protection against microorganisms, to enhance skin penetration, and as a solvent. In the present work, SEPINEO ${ }^{\mathrm{TM}}$ P 600 formulations and hydroxyethylcellulose gels containing the hydrophilic ILs hexylpyridinium chloride, choline dihydrogen phosphate, and 1-ethyl-3-methylimidazolium ethyl sulfate were prepared, and the influence of the ILs on the formulation properties was evaluated. ILs were successfully incorporated into the emulsion structure, resulting in stable formulations. The antimicrobial activity of the ILs was estimated. The minimal inhibitory concentration values for hexylpyridinium chloride are about $2.5 \mathrm{mg} / \mathrm{mL}$. The other two ILs have no antimicrobial activity. Skin penetration enhancement of caffeine, a hydrophilic model substance, was observed in the presence of hexylpyridinium chloride.
\end{abstract}

KEY WORDS: antimicrobial activity; emulsion; ionic liquids; skin penetration enhancement; surfaceactive agent.

\section{INTRODUCTION}

Controlled drug delivery through the skin is an alternative method of application for drugs that otherwise undergo quick metabolism and degradation in the gastrointestinal tract or liver after peroral administration or when local treatment of dermal and sub-dermal diseases is desired. The outermost skin layer, the stratum corneum, determines the barrier properties of the skin and prevents sufficient penetration of most drugs (1). Chemical skin permeation enhancers increase the transport across the barrier by partial solubilization or extraction of the skin lipids and by creating hydrophilic pores within the stratum corneum (2). However, permeation enhancers are often irritating and not well-tolerated (3). Aside from penetration enhancers, topical drug delivery systems contain several other potentially irritating ingredients including preservatives (4) and solvents. In this regard, it is of interest to identify new excipients that can replace potentially irritating compounds in dermal drug formulations.

\footnotetext{
${ }^{1}$ Institute of Bioprocess Engineering and Pharmaceutical Technology, Technische Hochschule Mittelhessen-University of Applied Sciences, Wiesenstr. 14, 35390, Giessen, Germany.

${ }^{2}$ Faculty of Biology and Chemistry, Justus Liebig University Giessen, Giessen, Germany.

${ }^{3}$ Pharmaceutical Technology and Biopharmacy, Philipps University Marburg, Marburg, Germany.

${ }^{4}$ To whom correspondence should be addressed. (e-mail: Dorota.Dobler@kmub.thm.de; Frank.Runkel@kmub.thm.de)
}

Ionic liquids (ILs) are organic salts that are in liquid form at temperatures less than $100^{\circ} \mathrm{C}$. They often replace conventional organic solvents because they have negligible vapor pressure, good chemical and thermal stability, high ionic conductivity, and the ability to dissolve both organic and inorganic materials (5). As designer solvents, ILs can be synthesized for particular applications or with specific chemical and physical properties simply by changing the anion/ cation combination $(6,7)$. Research on ILs in pharmaceutical applications has been of great interest in recent years. Ionic liquids are particularly useful for the solubilization of poorly soluble drugs (8). ILs can also be used to synthesize active pharmaceutical ingredients (APIs) with modified properties with regard to the starting materials (9-11). Furthermore, many ILs exhibit antimicrobial activity $(12,13)$, making them useful as APIs or formulation preservatives. Thus, ILs have several potential applications as additives in dermal formulations. However, only a few studies have been published regarding the use of ILs in pharmaceutical or cosmetic applications so far. ILs were used mostly in microemulsions (14) as oil substitutes, water substitutes, additives, or surfactants (15-17). Multiple emulsions containing ILs as a hydrophobic phase have also been reported (18). While hydrophilic ILs can be used to proportionally replace the water phase, hydrophobic ILs prefer to form an independent phase within the emulsion (19).

The aim of this study was to evaluate the functionality of different commercially available hydrophilic ionic liquids in dermal formulations. For this purpose, dermal drug delivery systems containing ILs were prepared and the influences of ILs on drug penetration and antimicrobial properties were studied. Furthermore, the effect of ILs on formulation properties was also investigated. 


\section{MATERIALS AND METHODS}

\section{Materials}

The oils used to prepare the delivery systems were ethylhexyl isononanoate (Cognis, Germany), medium-chain triglycerides (Caelo, Germany), and paraffin oil (Fagron, Germany). Cetostearyl alcohol (Evonic, Germany) was another emulsion component in the oil phase. The water phase contained glycerol (Fagron, Germany). Acrylamide/sodium acryloyldimethyl taurate copolymer/isohexadecane/polysorbate 80 (SEPINEO ${ }^{\text {тм }}$ P 600) (Seppic, France) was used as an emulsifying and gelling agent in SEPINEO ${ }^{\text {TM }} \mathrm{P} 600$ formulations. Hydroxyethylcellulose (Natrosol $250 \mathrm{HX}$ ) was purchased from Caelo.

Natrium chloride, orthophosphoric acid $85 \%$, sodium dihydrogen phosphate monohydrate, Mueller Hinton agar, Caso broth, potassium chloride, disodium hydrogen phosphate, and potassium dihydrogen phosphate were purchased from Carl Roth (Germany). Acetonitrile was obtained from VWR (Germany). Mueller Hinton broth was purchased from Fluka (Germany). Caffeine, 4-hydroxybenzoic acid propyl ester, and testosterone were purchased from Caelo (Germany). Igepal was supplied by Sigma-Aldrich (Germany).

The ionic liquids used were hexylpyridinium chloride [HPyr][Cl] $(\mathrm{MW}=199.73)$, choline dihydrogen phosphate [CDHP] (MW=201.16), and 1-ethyl-3-methylimidazolium ethyl sulfate [EMIM][EtSO4] (MW=236.29). The ILs were purchased by IoLiTec Ionic Liquids Technologies GmbH (Heilbronn, Germany) and have a purity $>98 \%$. The chemical structures of the ILs are shown in Fig. 1.

\section{Preparation of the Drug Delivery Systems}

Two different gels containing SEPINEO ${ }^{\text {TM }}$ P 600, three emulsion-gels containing SEPINEO ${ }^{\mathrm{TM}} \mathrm{P}$ 600, as well as one hydroxyethylcellulose (HEC) gel were prepared $(n=3)$. The IL concentrations in all formulations were 0,1 , and $5 \%$. The composition of tested formulations is presented in Table I.

SEPINEO ${ }^{\text {TM }} \mathrm{P} 600$ gels were prepared by mixing all components at $70^{\circ} \mathrm{C}$, followed by homogenization using a rotor/stator homogenizer (Diax 600, Heidolph Germany) for $1 \mathrm{~min}$ at 11,600 rpm. In the case of the SEPINEO ${ }^{\text {TM }} \mathrm{P} 600$ emulsions, the water phase and the oil phase were heated separately to $70^{\circ} \mathrm{C}$, and the two phases were mixed before homogenization under the same conditions.

HEC gels were prepared by mixing $2 \%$ of HEC in water containing dissolved ILs.

The formulation G2 was used for ex vivo skin permeation and drug uptake studies. As model drugs, the lipophilic testosterone and the hydrophilic caffeine were used. The concentrations of caffeine and testosterone in the formulation were $1 \mathrm{mg} / \mathrm{mL}$ and $200 \mu \mathrm{g} / \mathrm{mL}$, respectively. Drugs were dissolved in the water phase by stirring.

\section{Characterization of the Drug Delivery Systems}

Oil droplet size and size distribution in oil/water $(\mathrm{o} / \mathrm{w})$ emulsions were determined using a laser diffraction particle size analyzer (Mastersizer S, Malvern Instruments, UK). The fundamental size distribution obtained with this technique was based on the volume distribution. The particle size distribution was calculated according to the Mie theory. Rheological analysis of all formulations was performed at $25^{\circ} \mathrm{C}$ using a RheoStress 300 Rheometer (Thermo Haake) with a 2-cmdiameter cone and a plate geometry measuring system with a $2^{\circ}$ angle. The apparent viscosity was measured over a shear rate of $0.1-100 \mathrm{~s}^{-1}$. The $\mathrm{pH}$ of the formulations was determined using a portable Mettler Toledo system with a glass InLab Viscous $\mathrm{pH}$ electrode. All results are presented as mean values and standard deviations of three independent experiments.

\section{Surface Tension and Conductivity Measurements of the Water Solution}

Surface tension measurements of water containing ILs were made at $20^{\circ} \mathrm{C}$ using the du Noüy ring method and a TD1-M tensiometer (Lauda, Germany). The conductivity of water containing ILs was measured at $20^{\circ} \mathrm{C}$ using a WTW Microprocessor Conductivity Meter LF 96 with a Tetracon 325 electrode (WTW, Germany).

\section{Determination of the Antimicrobial and Antifungal Activities}

Antibacterial and antifungal activities of the ILs were evaluated through determination of the minimal inhibitory concentrations (MIC) using the microdilution method in a 96-well microplate. IL-containing tubes were inoculated with a standardized bacterial suspension of $1-5 \times 10^{5} \mathrm{CFU} / \mathrm{mL}$ in Mueller Hinton broth medium. The reference strains used were Staphylococcus gallinarum, Escherichia coli, and Kluyveromyces marxianus. Dilution series of the ionic liquids were prepared from 50 to $0.009 \mathrm{mg} / \mathrm{mL}$ in sterile distilled water. Positive (containing parabene as preservatives) and negative (no treatment) growth controls were included in each assay. After incubation for $24 \mathrm{~h}$ at $37^{\circ} \mathrm{C}$, the lowest concentration free from turbidity was set as the MIC. The turbidity was determined using a microplate absorbance reader (Sunrise Plate reader, Tecan) at $600 \mathrm{~nm}$.<smiles>C[N+](C)(C)CCO</smiles><smiles>CCCCCC[n+]1ccccc1</smiles><smiles>CCOS(=O)(=O)[O-]</smiles>

Fig. 1. Structure of ionic liquids used for the experiments. a choline dihydrogen phosphate, b hexylpyridinium chloride, and $\mathbf{c}$ 1-ethyl-3-methylimidazolium ethyl sulfate 
Table I. Composition of all Formulations $(n=3)$. Oils: E1-Medium-Chain Triglycerides, E2-Paraffin Oil, or E3-Ethylhexyl Isononanoate

\begin{tabular}{|c|c|c|c|c|c|}
\hline & & $\begin{array}{l}\text { SEPINEO } \\
\text { gel } 1, \mathrm{G} 1(\mathrm{wt} \%)\end{array}$ & $\begin{array}{l}\text { SEPINEO } \\
\text { gel } 2, \mathrm{G} 2(\mathrm{wt} \%)\end{array}$ & $\begin{array}{l}\text { SEPINEO }{ }^{\mathrm{TM}} \text { emulsions } \\
1-3, \text { E1-E3 (wt } \%)\end{array}$ & $\begin{array}{r}\text { HEC gel } \\
(w t \%)\end{array}$ \\
\hline \multirow[t]{3}{*}{ Lipophilic phase } & Cetostearyl alcohol & - & - & 1.5 & - \\
\hline & SEPINEO $^{\text {Tм }}$ P 600 & - & - & 4.0 & - \\
\hline & Oil & - & - & 15.0 & - \\
\hline \multirow[t]{6}{*}{ Hydrophilic phase } & Cetostearyl alcohol & - & 1.5 & - & - \\
\hline & SEPINEO ${ }^{\text {TM }}$ P 600 & 4.0 & 4.0 & - & - \\
\hline & Ionic liquid & $0.0 / 1.0$ or 5.0 & $0.0 / 1.0$ or 5.0 & $0.0 / 1.0$ or 5.0 & $0.0 / 1.0$ or 5.0 \\
\hline & Glycerol 85\% & 3.0 & 3.0 & 3.0 & - \\
\hline & HEC & - & - & - & 2 \\
\hline & Distilled water & Ad 100 & Ad 100 & Ad 100 & Ad 100 \\
\hline
\end{tabular}

\section{Solubility Measurements}

4-Hydroxybenzoic acid propyl ester and testosterone were used as model water-insoluble pharmaceutical compounds. Solubility was measured by mixing $200-400 \mathrm{mg}$ of substance with $1 \mathrm{~g}$ IL or $1 \mathrm{~g}$ IL/water solution overnight at $30^{\circ} \mathrm{C}$. After that, the samples were incubated for 24 and $72 \mathrm{~h}$ at $25^{\circ} \mathrm{C}$. The mixtures were immediately centrifuged and the supernatant was filtered (pore size $0.45 \mu \mathrm{m}$ ) and analyzed by HPLC.

\section{Skin Irritation and Corrosion Tests}

The skin irritation and skin corrosion studies were conducted in accordance with OECD Guideline for the Testing of Chemicals $(20,21)$. The test IL remained undiluted and was applied topically to a human reconstructed epidermis model (EpiDerm ${ }^{\mathrm{TM}}$ ). After specified exposure periods, the cell viability was determined using the MTT cell viability test.

\section{Ex Vivo Skin Permeation and Drug Uptake Studies}

Skin penetration assays were performed ex vivo using the Franz diffusion cell setup (22). As model drugs, lipophilic testosterone $(\log \mathrm{P}=3.47)$ and hydrophilic caffeine $(\log \mathrm{P}=-0.013)$ were used. Porcine skin was chosen due to its similarity to human skin in terms of its morphology and permeability characteristics (23) and due to its availability. Porcine ears were washed by rinsing with mildly warm water and were wiped with paper towels; the bristles were carefully shortened by trimming. Full-thickness porcine skin was stored at $-20^{\circ} \mathrm{C}$ (up to 6 months) and thawed prior to use. On the day of experiment, the prepared skin samples were mounted in the Franz diffusion cell with $\mathrm{PBS} \mathrm{pH}=7.4$ (or PBS containing 0.5\% Igepal, Sigma-Aldrich, for penetration of testosterone) as the acceptor fluid (tempered at $32.5^{\circ} \mathrm{C}$ ) and the cells were equilibrated for $30 \mathrm{~min}$. The skin diffusion area was $1.76 \mathrm{~cm}^{2}$. These experimental conditions have been previously verified (24).

Then, the $500 \mu \mathrm{L}$ (infinite dose) of the investigated formulations (SEPINEOTM gel 2) was evenly applied onto the skin surface for $24 \mathrm{~h}$. At the end of the experiment, any remaining formulation was removed by a wash-off procedure.
The skin samples, acceptor fluid, and supernatant were analyzed by HPLC.

\section{HPLC Analysis}

HPLC analysis was performed on a VWR-HITACHI chromatographic system (Germany) consisting of an L2130 pump equipped with an L2200 autosampler and column heater set and a L2455 photodiode array detector. A RP-C18 LiChrospher $1005-\mu \mathrm{m}$ column was used. EZChrome EliteClient software version 3.1.7 was used for peak integration. The equipment was operated at $40^{\circ} \mathrm{C}$. The methods for HPLC analysis of caffeine and testosterone have been described in detail elsewhere (24).

\section{RESULTS}

\section{Surface Tension and Conductivity Measurements}

The behavior of ionic liquids in aqueous media was determined by surface tension and conductivity measurements. Using these methods, the degree of dissociation, the surface activity, and the micelle formation concentration of ILs can be detected. Figure 2 shows the surface tension and conductivity as a function of the IL concentration. The concentrations correspond to the concentrations used in tested formulations (up to $5 \mathrm{wt} \%$ ).

With increasing IL concentration, a linear increase in conductivity was observed for all ILs. The strongest increase was observed for [HPyr][Cl]. If an abrupt change of slopes is observed, the corresponding value can be derived for the critical aggregate concentration (CAC) (25). This behavior was not observed for tested ILs (Fig. 2a). The surface tension of the $[\mathrm{HPyr}][\mathrm{Cl}]$ solution decreased more with increasing IL concentration, when compared to [CDHP] and [EMIM][EtSO4]. However, a minimum surface tension, which indicates the CAC, was not found. A change in surface tension was not detected for [CDHP] and [EMIM][EtSO4] at the concentrations analyzed.

\section{Formulation Characterization}

Two different gels and three gel-emulsions containing SEPINEOTM $\mathrm{P} 600$ were produced. The viscosity and $\mathrm{pH}$ value of SEPINEO ${ }^{\text {тм }} \mathrm{P} 600$ formulations were measured one day after preparation (Fig. 3). 

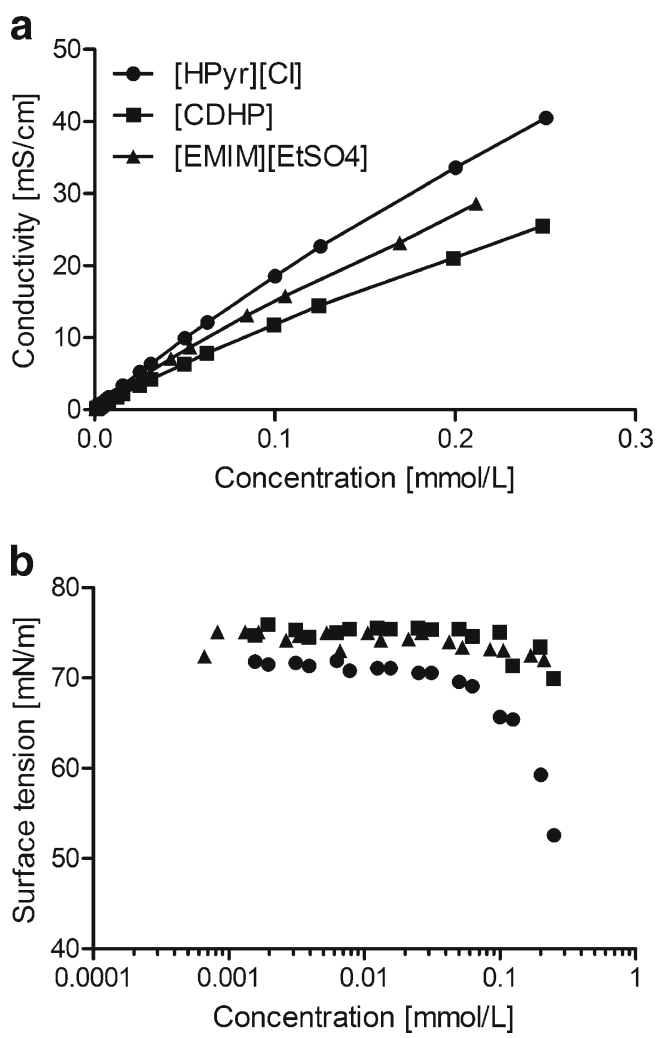

Fig. 2. Plots of a conductivity and $\mathbf{b}$ surface tension vs IL concentration in water for the examined ILs: [HPyr][Cl], [CDHP], and [EMIM][EtSO4] (mean value $\pm \mathrm{SD}, n=3$ )

The viscosity of the formulations without ILs is dependent on their composition. The formulations without oil phase (G1 and G2) are defined as gels. Increase in viscosity of G2 compared to G1 is connected with the addition of a thickening agent, cetylstearyl alcohol, which leads to a creation of a liquid crystalline phase (26). Addition of the oil phase in formulation $\mathrm{E}$ (E1-E3) leads mostly to an increase in viscosity of up to $\sim 6 \mathrm{~Pa}$ s. In this case, a gel-emulsion texture is obtained. The viscosity of the gel-emulsions E1-E3 depends on the used oil. Emollients, such as ethylhexyl isononanoate, generated emulsions with a lower viscosity $(3.10 \pm 0.02 \mathrm{~Pa} \mathrm{~s})$ than paraffin $(5.72$ $\pm 0.02)$ and medium-chain triglycerides $(6.28 \pm 0.07)$. The $\mathrm{pH}$ values of all formulations without ILs, independent of composition, indicate that they are slightly acidic $(\mathrm{pH} \approx 5.5)$ (data for $\mathrm{G} 1$ are presented in Fig. 3b). The droplet sizes $(D(0,5))$ of gel-emulsion formulations were $3.0 \pm 0.3,4.8 \pm 0.4$, and 3.5 $\pm 0.3 \mu \mathrm{m}$, respectively.

The addition of the hydrophilic ILs resulted in a strong decrease of viscosity, which is concentration-dependent and almost independent of the used IL. The strongest decrease of viscosity is observed for the G1 formulation, while the gelemulsions (E1-E3) remain viscous. The $\mathrm{pH}$ value of all formulations also is IL-concentration-dependent. In contrast to viscosity, $\mathrm{pH}$ value also depends strongly on the used IL. [CDHP] slightly decreased the $\mathrm{pH}$ of all formulations (down to $\mathrm{pH} \approx 5)$, as compared to the formulation without IL (Fig. 3b). The major alteration in $\mathrm{pH}$ was observed for [EMIM][EtSO4]. At 5\% of [EMIM][EtSO4], a pH value of about 2.5 was measured. No significant change in droplet sizes compared to the gel-emulsions without ILs was observed.

A second type of dermal formulation, a HEC gel, was prepared. The formulations without ILs had a viscosity of about $2 \mathrm{~Pa} \mathrm{~s}$ and $\mathrm{pH}$ values of about 6.5. The addition of ILs resulted in a decrease of $\mathrm{pH}$ values similar to that of the SEPINEO ${ }^{\mathrm{TM}} \mathrm{P} 600$ formulations. In contrast to SEPINEO ${ }^{\mathrm{TM}} \mathrm{P} 600$ gels, ILs did not influenced the viscosity of HEC gels.

All observed formulations were stable over the time of storage (3 months, data not shown), i.e., no phase separation and no significant changes in viscosity, droplet size, or $\mathrm{pH}$ values were observed.

\section{Determination of Antimicrobial Activity}

Gram-positive bacteria ( $S$. gallinarum), gram-negative bacteria ( $E$. coli), and one strain of fungi ( $K$. marxianus) were used to assess the antimicrobial activity of the ILs. The results of the turbidity measurements are presented in Fig. 4.

A substantial decrease in turbidity correlating to the inhibition of microorganism growth (survival) was observed for $[\mathrm{HPyr}][\mathrm{Cl}]$. The MIC value determined for all tested bacteria and fungi is $2.5 \mathrm{mg} / \mathrm{mL}$. The inhibition effect in the presence of [EMIM][EtSO4] occurs at significantly higher concentrations, and MIC values were not obtained for the concentrations analyzed. The bacteria behavior/growth in the presence of [CDHP] was clearly different, as an increase in turbidity was observed, indicating faster bacteria growth.

\section{Solubility Determination}

The solubility of 4-hydroxybenzoic acid propyl ester and testosterone in various ILs, as well as in water solutions of hydrophilic ILs, is shown in Table II. Because of melting temperatures above room temperature, [CDHP] and [Py6] [Cl] were tested only in water solutions. ILs containing imidazolium- or pyridinium-based cations are found to be very good solvents for the tested drugs. The solubility of tested substances in $[\mathrm{HPyr}][\mathrm{Cl}]$ solution was found to be greater than in [EMIM][EtSO4]. No enhanced solubility of the tested drugs in $[\mathrm{CDHP}]$ was detected.

\section{Skin Irritation and Skin Corrosion Tests}

The most toxic IL ([HPyr][Cl]) (27-29) was tested with regard to its irritation and corrosion potential. It could be shown that undiluted $[\mathrm{HPyr}][\mathrm{Cl}]$ is irritative, but not corrosive. For both tests, cell viability of the skin tissue was evaluated using an MTT test. In the skin corrosion test, after 3 min of treatment with $[\mathrm{HPyr}][\mathrm{Cl}]$, the relative absorbance values were reduced to $91.4 \%$. This value is well above the threshold for corrosion potential $(50 \%)$. After $1 \mathrm{~h}$ of treatment, the relative absorbance values were reduced to $69.6 \%$. This value is also well above the corrosion potential (15\%).

Regarding the skin irritation test, treatment with $[\mathrm{HPyr}][\mathrm{Cl}]$ resulted in a reduction of the relative absorbance values to $15.7 \%$. This value is well below the threshold for irritation potential $(50 \%)$. As a positive control for this 

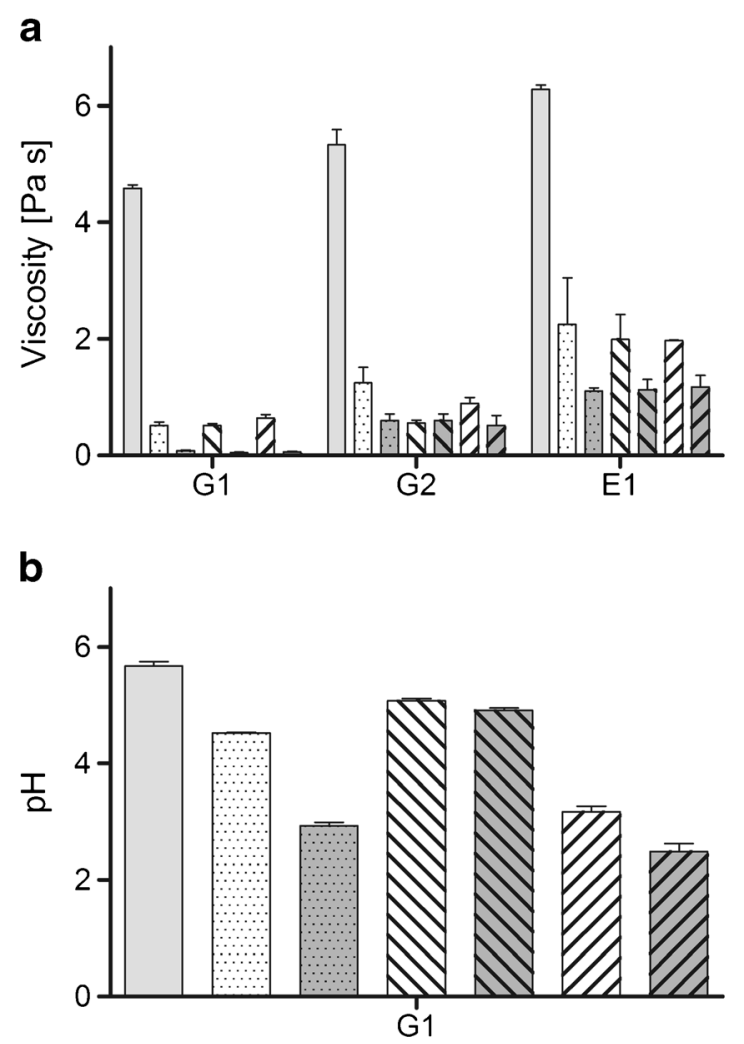

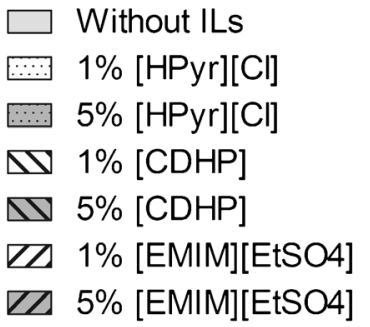

Fig. 3. a Viscosity and $\mathbf{b} \mathrm{pH}$ of SEPINEO ${ }^{\mathrm{TM}} \mathrm{P} 600$ formulations containing different ILs: [HPyr][Cl], [CDHP], and [EMIM][EtSO4]. The formulation designation is in accordance to Table I: G1 SEPINEO ${ }^{\mathrm{TM}}$ gel 1, G2 SEPINEO ${ }^{\mathrm{TM}}$ gel 2, E1 SEPINEO ${ }^{\mathrm{TM}}$ emulsion 1 (mean value $\pm \mathrm{SD}, n=3$ )

experiment, a 5\% solution of SDS (sodium dodecyl sulfate) was used. It is an anionic surfactant used in many cleaning and hygiene products (in concentrations above 5\%). The irritation potential, expressed as cell viability, was lower for undiluted [HPyr $][\mathrm{Cl}]$ (cell viability $15.7 \%$ ) than for a $5 \%$ solution of SDS (cell viability $2 \%$ ). Therefore, it can be supposed that $[\mathrm{HPyr}][\mathrm{Cl}]$ is significantly less irritating than commonly used ionic surfactants.

\section{Ex Vivo Skin Permeation and Drug Uptake Studies}

The skin penetration and permeation $(24 \mathrm{~h})$ of caffeine and testosterone were performed with the gel formulation G2 containing $5 \%[\mathrm{HPyr}][\mathrm{Cl}]$ and were compared to the same formulation without IL (Table III). Caffeine uptake from the gel formulation $\mathrm{G} 2$ containing [HPyr] $[\mathrm{Cl}]$ was twofold higher after a 24-h period, compared to the formulation without $[\mathrm{HPyr}][\mathrm{Cl}]$. No testosterone was detected in the acceptor medium under this condition.

\section{DISCUSSION}

The used ILs were selected due to their good water solubility (above $5 \mathrm{wt} \%$ ), low to moderate toxicity $(28,29)$, and relatively low irritation potential. Furthermore, the ILs can be used as solvents for pharmaceutical components (30) and choline salts can be useful for stabilization of protein-based APIs in the formulation (31). [HPyr] $[\mathrm{Cl}]$ and [CDHP] are solid at RT, whereas [EMIM][EtSO4] is a highly viscous fluid.
Formulations that included SEPINEO ${ }^{\text {TM }} \mathrm{P} 600$ resulted in high-viscosity gels or emulsion-gels. Addition of cetylstearyl alcohol as an additional thickener led to a slight increase in viscosity, in comparison to the formulation without cetylstearyl alcohol, due to the formation of a liquid crystalline phase (32). A further increase in viscosity was observed after adding mediumchain triglycerides or paraffin oil as an oil phase.

SEPINEO ${ }^{\mathrm{TM}} \mathrm{P}$ 600, which is used in the present study, has self-gelling and thickening properties and the ability to emulsify an oil phase. In gels, the system rheology is dependent upon the SEPINEO ${ }^{\mathrm{TM}} \mathrm{P} 600$ concentration. In the emulsion-gel containing an oil phase, the rheological characteristic is affected by additional interactions between oil droplets and the gel matrix (33). Oil droplets increase the SEPINEO ${ }^{\text {TM }} \mathrm{P} 600$ concentration in the water phase of the emulsion, and the latter strengthens the gel network, resulting in higher viscosity values. The chemical characteristics of oils used, particularly the viscosity, spreadability, and polarity of the oil, as well as its compatibility with other emulsion ingredients, influence the structure of the formulation, resulting in different rheological parameters (34). However, due to the complex interactions, no exact correlation between oil properties and the viscosity of the emulsion could be derived.

The addition of ILs strongly influences the viscosity of the SEPINEO ${ }^{\text {TM }} \mathrm{P} 600$ formulations. It is known that the surface activity and the ionic character of the components dissolved in the water phase have an important influence on the parameters of the formulation. ILs investigated in this paper exhibited characteristics of short-chain surface-active substances. The alkyl chains have six ([HPyr][Cl]), two ([EMIM][EtSO4]), or one 


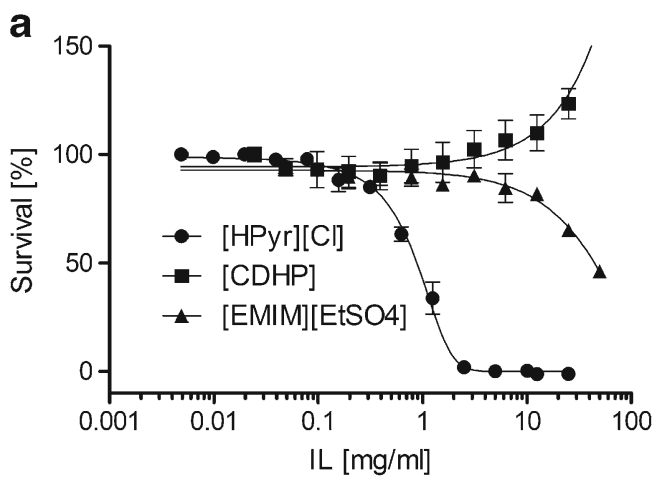

b
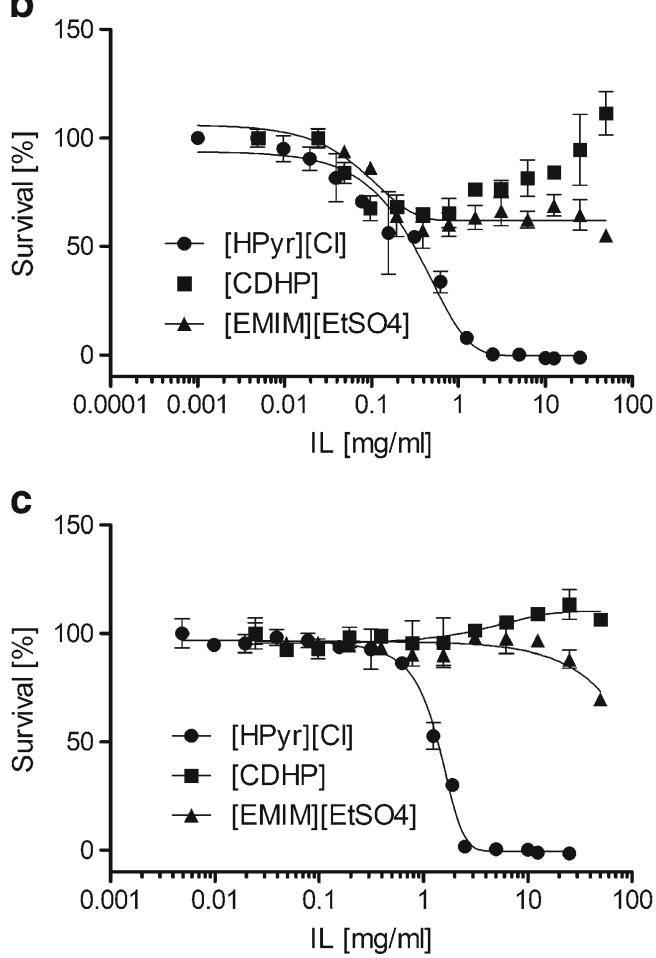

Fig. 4. Effect of the ILs ([HPyr][Cl], [CDHP], and [EMIM] [EtSO4]) on survival of bacterial cells measured as \% survival of bacteria in the presence of ILs compared to positive control ( $0.2 \%$ parabene) for a Escherichia coli, b Staphylococcus gallinarum, and c Kluyveromyces marxianus by turbidity measurement at $600 \mathrm{~nm}$ (mean value $\pm \mathrm{SD}$, $n=3)$

([CDHP]) carbon atom(s). Therefore, low surface activity and $\mathrm{CAC}$ values at high concentrations, or no aggregation at all is expected. The surface-active substances can act as an emulsifier.
Additionally, in the case of the formation of micelles, some ingredients can be enclosed resulting in destabilization of the formulation. For [HPyr][Cl], the constant decrease in surface tension, as well as the linear increase in conductivity at the concentrations analyzed, suggests distinctive surface activity but no creation of aggregates (micelles). Based on the data, it can be assumed that $[\mathrm{HPyr}][\mathrm{Cl}]$ can act as an emulsifier with cationic character. The low surface activity of [CDHP] and [EMIM][EtSO4] indicates that these ILs will preferably not act as surfactants and will not create aggregates. The observed decrease of viscosity of the SEPINEO ${ }^{\mathrm{TM}} \mathrm{P} 600$ formulations is independent of the IL used. Therefore, the connection between the surface activity and the viscosity is not fully explained. For the presented formulations, it is likely that the ionic character of the ILs is responsible for the observed alteration of the viscosity. Cationic surfactants and other cationic ingredients can also interact with gelling agents such as polyacrylic acid. They are absorbed into the gel, which leads to gel shrinkage and results in fall in viscosity (35). Nevertheless, despite the reduced viscosity, all samples remained stable over time.

In contrast to SEPINEO ${ }^{\mathrm{TM}} \mathrm{P} 600$ formulations, a decrease in viscosity in the presence of ILs was not observed for HEC gels. However, it is known that the properties (especially viscosity) of the HEC systems can be improved by adding small amounts of surfactants or ionic substances. The primary attractive forces between HEC and the added species are hydrophobic interactions. Furthermore, the presence of oppositely charged species enhances the interaction (36). In the present work, hydrophobically unmodified nonionic HEC was used. The interaction between the polymer and ionic substances is thus weak. Therefore, no significant alternation of the viscosity was expected.

The differences in the $\mathrm{pH}$ values of SEPINEO ${ }^{\mathrm{TM}} \mathrm{P} 600$ and HEC formulations in the presence of ILs are connected with their chemical character. The majority of ions used in the formulation of ionic liquids can be considered in terms of the Lewis/ Brønsted definition of acids and bases. The resulting character of the IL is thus directly connected to the character of both ions. The $\mathrm{pH}$ of ILs can also be influenced significantly by small amounts of impurities (37). Typical impurities are organic starting materials, halide, protic impurities, and other ionic impurities from incomplete metathesis reactions. Due to the slight acidic or neutral character of the ions used in this experiment, the substantial decrease in $\mathrm{pH}$ is most likely a result of impurities.

The ILs as additives in dermal formulations can have different functions. These include antimicrobial activity and the enhancement of skin penetration of the active ingredients. The antimicrobial activity of ILs is primarily dependent upon the length of the alkyl chain of the cation. The most active

Table II. Solubility of 4-Hydroxybenzoic Acid Propyl Ester and Testosterone in ILs (mg/mL, Mean Value \pm SD, $n=3$ )

\begin{tabular}{lll}
\hline $\mathrm{IL}$ & 4-Hydroxybenzoic acid propyl ester & Testosterone \\
\hline$[\mathrm{CDHP}] / \mathrm{H}_{2} \mathrm{O}(50: 50 w / w)$ & $0.11 \pm 0.01$ & $0.001 \pm 0.001$ \\
{$[\mathrm{CDHP}] / \mathrm{H}_{2} \mathrm{O}(25: 75 w / w)$} & $0.16 \pm 0.01$ & 0 \\
{$[\mathrm{HPyr}][\mathrm{Cl}] / \mathrm{H}_{2} \mathrm{O}(50: 50 \mathrm{w} / \mathrm{w})$} & $167.16 \pm 1.70$ & $3.96 \pm 0.09$ \\
{$[\mathrm{HPyr}][\mathrm{Cl}] / \mathrm{H}_{2} \mathrm{O}(25: 75 \mathrm{w} / \mathrm{w})$} & $97.66 \pm 0.63$ & $1.70 \pm 0.08$ \\
{$[\mathrm{EMIM}][\mathrm{EtSO} 4]$} & $199.81 \pm 1.38$ & $33.47 \pm 0.91$ \\
{$[\mathrm{EMIM}][\mathrm{EtSO} 4] / \mathrm{H}_{2} \mathrm{O}(50: 50 w / w)$} & $12.79 \pm 0.16$ & $0.34 \pm 0.06$ \\
{$[\mathrm{EMIM}][\mathrm{EtSO} 4] / \mathrm{H}_{2} \mathrm{O}(25: 75 w / w)$} & $3.92 \pm 0.07$ & $0.14 \pm 0.01$ \\
Water & 0.5 & 0.02 \\
\hline
\end{tabular}


Table III. Results of Caffeine and Testosterone Skin Uptake Study of Gel Formulation G2, with and without [HPyr][Cl] After 24 h (mean value $\pm \mathrm{SD}, n=3$ )

\begin{tabular}{|c|c|c|c|c|}
\hline & \multicolumn{2}{|l|}{ Caffeine } & \multicolumn{2}{|l|}{ Testosterone } \\
\hline & $\begin{array}{l}\text { Formulation without } \\
{[\mathrm{HPyr}][\mathrm{Cl}]}\end{array}$ & $\begin{array}{l}\text { Formulation with } \\
5 \%[\mathrm{HPyr}][\mathrm{Cl}]\end{array}$ & $\begin{array}{l}\text { Formulation without } \\
{[\mathrm{HPyr}][\mathrm{Cl}]}\end{array}$ & $\begin{array}{l}\text { Formulation with } \\
5 \%[\mathrm{HPyr}][\mathrm{Cl}]\end{array}$ \\
\hline Flux $\left(\mu \mathrm{g} / \mathrm{cm}^{2} / \mathrm{h}\right)$ & $1.93 \pm 0.05$ & $2.63 \pm 0.5$ & - & - \\
\hline Drug uptake $^{\mathrm{a}}(\%)$ & $7.70 \pm 0.3$ & $13.80 \pm 0.7$ & 0 & 0 \\
\hline Drug in the skin ${ }^{\mathrm{b}}(\%)$ & $0.80 \pm 0.1$ & $2.50 \pm 0.3$ & $0.07 \pm 0.02$ & $0.07 \pm 0.01$ \\
\hline Total recovery ${ }^{\mathrm{b}}(\%)$ & $97.90 \pm 1.3$ & $102.90 \pm 2.4$ & $101.40 \pm 1.9$ & $98.90 \pm 0.3$ \\
\hline
\end{tabular}

${ }^{a}$ Total drug uptake: drug in the skin plus drug in the acceptor medium after $24 \mathrm{~h}$

${ }^{b}$ Percentage of the applied drug amount

salts have alkyl chain lengths between 10 and 14 carbon atoms (13). Accordingly, the highest activity for ILs analyzed in this study was observed for [HPyr] $[\mathrm{Cl}]$ with a six-C-atom alkyl chain. [EMIM][EtSO4] (two carbon atoms) exhibits a low inhibitory activity at concentrations above $50 \mathrm{mg} / \mathrm{mL}$. In contrast, [CDHP] seems to stimulate the growth of the bacteria. Secar et al. observed a similar result with bacteria in the presence of choline salts. They concluded that biocompatible choline salts can be used as the carbon source needed for nutrition of some microorganisms (38). Based on this data, $[\mathrm{HPyr}][\mathrm{Cl}]$ may only be used as an antimicrobial agent or as preservative. However, the MIC value of [HPyr] [Cl] $(2.5 \mathrm{mg} /$ $\mathrm{mL}$ ) is significantly higher than the MIC values of commonly used preservatives (Table III). The advantage of $[\mathrm{HPyr}][\mathrm{Cl}]$ compared to common preservatives is its water solubility (Table IV). Important factors affecting the activity of preservatives in $\mathrm{o} / \mathrm{w}$ emulsions are those controlling the availability of the preservative in the aqueous phase and, in particular, the $\mathrm{o} / \mathrm{w}$ partition coefficient of the preservative. Poorly soluble preservatives, such as $p$-hydroxybenzoic acid esters, diffuse predominantly in the oil phase where they are not active against microorganisms. Therefore, in systems with oil phases, higher concentrations of preservatives are necessary. In contrast, water-soluble substances do not require an excess dosage for sufficient preservation.

The solubility of drugs in ILs is strongly dependent on the specific IL, in that the structure of cations as well as anions is of importance. The good solvent properties of ILs containing imidazolium- or pyridinium-based cations with regard to drugs containing aromatic rings are already shown in the literature (42). In this case, hydrogen bonds, van der Waal's forces, and $\pi-\pi$ interactions between aromatic rings are likely involved in drug-solvent interactions (43). Solubility in a $[\mathrm{HPyr}][\mathrm{Cl}]$ solution is found to be greater than in [EMIM][EtSO4], which is connected to alkyl chain length. Due to increasing hydrophobic character, the longer the alkyl chain, the greater is the solvency for the hydrophobic drugs (42). Good solvent properties were also found in the mixtures of water and ILs, which indicated a persistent solvent-drug interaction. In the waterCDHP solution, the solubility of tested drugs was even lower than in pure water. This was probably due to the strong hydrophilic and polar character of CDHP.

Penetration enhancers may be incorporated into formulations in order to improve drug flux through different membranes, including the skin membrane. Diffusion through skin is controlled by the outermost layer, the stratum corneum (SC), and can be regarded as diffusion through a passive membrane. A penetration enhancer may act as follows: increase the diffusion coefficient of the drug through the stratum corneum, increase the effective concentration of the drug in the vehicle (for example, acting as an anti-solvent), or improve partitioning between the formulation and the stratum corneum (by altering the solvent nature of the skin membrane to improve partitioning into the tissue) (44). The positive effect of ILs on penetration is related to their ionic character and their property to act as cationic surfactants. Ionic surfactants can penetrate the stratum corneum membrane and, by extracting the lipids from the SC, disrupt the lipid bilayer packing within the tissue (44). As a result, other substances can more easily penetrate the skin; however, the penetration enhancement is still dependent upon the chemical character of the drug substance. A hydrophilic substance cannot penetrate the skin easily, because it cannot

Table IV. Properties of Commonly Used Preservatives in Comparison to [HPyr][Cl]

\begin{tabular}{lccc}
\hline & LogP & Water solubility $(\mathrm{g} / \mathrm{L})$ & $\mathrm{MIC}(\mathrm{mg} / \mathrm{mL})$ \\
\hline$[\mathrm{HPyr}][\mathrm{Cl}]$ & $-1.89^{\mathrm{a}}$ & $>100^{\mathrm{a}}\left(20^{\circ} \mathrm{C}\right)$ & $2.5^{\mathrm{a}}$ \\
$p$-Hydroxybenzoic acid methyl ester & 1.96 & $2.5\left(25^{\circ} \mathrm{C}\right)$ & $0.5-4.0^{\mathrm{b}}$ \\
$p$-Hydroxybenzoic acid propyl ester & 3.04 & $0.5\left(25^{\circ} \mathrm{C}\right)$ & $0.04-1.0^{\mathrm{b}}$ \\
Sorbic acid & 1.33 & $1.6\left(20^{\circ} \mathrm{C}\right)$ & $0.025-0.5^{\mathrm{c}}$ \\
Phenoxyethanol & 1.16 & $30\left(20^{\circ} \mathrm{C}\right)$ & $0.6-15^{\mathrm{d}}$ \\
\hline
\end{tabular}

\footnotetext{
${ }^{a}$ Intern study

${ }^{b}$ Aalto et al. (39)

${ }^{c}$ Lück and Jager (40)

${ }^{d}$ Lundov et al. (41)
} 
enter the lipophilic SC layer. In contrast, a lipophilic substance can more easily enter the SC, but it remains inside the SC because the lower layer, the dermis, has a more hydrophilic character. The [HPyr][Cl] allowed higher penetration of hydrophilic caffeine into the skin, which resulted in a higher permeation. This indicates that the lipophilic SC layer had been modified by [HPyr] $[\mathrm{Cl}]$, thus making it more permeable for hydrophilic substances. In contrast, penetration enhancement by $[\mathrm{HPyr}][\mathrm{Cl}]$ was not observed for lipophilic testosterone.

\section{CONCLUSION}

The results obtained in this experimental series highlight the suitability of ILs as ingredients of dermal formulations. However, due to their ionic character, potential incompatibilities of ILs with formulation ingredients must be evaluated. The conductivity and surface activity of several hydrophilic ionic liquids in a water solution were determined. Based on the data, SEPINEO ${ }^{\text {TM }}$ P 600 gels and emulsion-gels as well as HEC gels were produced. All ILs evaluated disturbed the gel structure of SEPINEO ${ }^{\text {TM }} \mathrm{P} 600$ gels, resulting in a decrease in viscosity. They are absorbed similar to cationic surfactants into the gel, leading to gel shrinkage and fall in viscosity. However, long-term stability of the formulations was not affected. All emulsion parameters remained unchanged during storage. In contrast, the viscosity of the HEC gels was not affected by the addition of the ILs.

Depending on the chemical structure of an IL, it can provide one or more functions of conventional excipients. These include protection against microorganisms, penetration enhancement, and solvent function. The antimicrobial activity of ILs is strongly dependent on their chemical structure. Because of its long alkyl chain, the antimicrobial activity of [HPyr] $[\mathrm{Cl}]$ is significantly higher than the activity of [CDHP] and [EMIM][EtSO4]. With MIC values of approximately $2.5 \mathrm{mg} /$ $\mathrm{mL},[\mathrm{HPyr}][\mathrm{Cl}]$ can be successfully used as a preservative in formulations. Additionally, penetration enhancement of hydrophilic caffeine in the presence of $[\mathrm{HPyr}][\mathrm{Cl}]$ was observed.

\section{ACKNOWLEDGMENTS}

This project (HA project no. 363/13-02) is funded in the framework of Hessen Modell Projekte, financed with funds of LOEWE-Landes-Offensive zur Entwicklung Wissenschaftlich-ökonomischerExzellenz, Förderlinie 3: KMU-Verbundvorhaben (State Offensive for the Development of Scientific and Economic Excellence). Furthermore, we would like to acknowledge the professional support and cooperation of Biodermic Health \& Beauty GmbH \& Co. KG. Biodermic managed all regulatory and business belongings in the project, making the ILs a marketable cosmetic product.

\section{REFERENCES}

1. Scheuplein RJ, Blank IH. Permeability of the skin. Physiol Rev. 1971;51(4):702-47.

2. Cevc G. Drug delivery across the skin. Expert Opin Investig Drugs. 1997;6(12):1887-937. Epub 2005/07/02.

3. Sasaki H, Kojima M, Nakamura J, Shibasaki J. Acute toxicity and skin irritation of pyrrolidone derivatives as transdermal penetration enhancer. Chem Pharm Bull (Tokyo). 1990;38(8):2308-10.
4. Lee E, An S, Choi D, Moon S, Chang I. Comparison of objective and sensory skin irritations of several cosmetic preservatives. Contact Dermatitis. 2007;56(3):131-6.

5. Olivier-Bourbigou H, Magna L, Morvan D. Ionic liquids and catalysis: recent progress from knowledge to applications. Appl Catal A Gen. 2010;373(1-2):1-56.

6. Zhang S, Sun N, He X, Lu X, Zhang X. Physical properties of ionic liquids: database and evaluation. J Phys Chem Ref Data. 2006;35(4):1475-517.

7. Tokuda H, Ishii K, Susan MA, Tsuzuki S, Hayamizu K, Watanabe M. Physicochemical properties and structures of roomtemperature ionic liquids. 3. Variation of cationic structures. J Phys Chem B. 2006;110(6):2833-9. Epub 2006/02/14.

8. Moniruzzaman M, Goto M. Ionic liquids: future solvents and reagents for pharmaceuticals. J Chem Eng Jpn. 2011;44(4):370-81.

9. Bica K, Rijksen C, Nieuwenhuyzen M, Rogers RD. In search of pure liquid salt forms of aspirin: ionic liquid approaches with acetylsalicylic acid and salicylic acid. Phys Chem Chem Phys. 2010;12(8):2011-7.

10. Bica K, Rodriguez H, Gurau G, Cojocaru OA, Riisager A, Fehrmann R, et al. Pharmaceutically active ionic liquids with solids handling, enhanced thermal stability, and fast release. Chem Commun (Camb). 2012;48(44):5422-4. Epub 2012/04/27.

11. Hough WL, Rogers RD. Ionic liquids then and now: from solvents to materials to active pharmaceutical ingredients. Bull Chem Soc Jpn. 2007;80(12):2262-9.

12. Iwai N, Nakayama K, Kitazume $\mathrm{T}$. Antibacterial activities of imidazolium, pyrrolidinium and piperidinium salts. Bioorg Med Chem Lett. 2011;21(6):1728-30. Epub 2011/02/18.

13. Pernak J, Sobaszkiewicz K, Mirska I. Anti-microbial activities of ionic liquids. Green Chem. 2003;5(1):52-6.

14. Moniruzzaman M, Tahara Y, Tamura M, Kamiya N, Goto M. Ionic liquid-assisted transdermal delivery of sparingly soluble drugs. Chem Commun (Camb). 2010;46(9):1452-4. Epub 2010/02/18.

15. Qiu ZM, Texter J. Ionic liquids in microemulsions. Curr Opin Colloid Interface Sci. 2008;13(4):252-62.

16. Zech O, Thomaier S, Bauduin P, Ruck T, Touraud D, Kunz W. Microemulsions with an ionic liquid surfactant and room temperature ionic liquids as polar pseudo-phase. J Phys Chem B. 2009;113(2):465-73. Epub 2008/12/23.

17. Li J, Zhang J, Gao H, Han B, Gao L. Nonaqueous microemulsion-containing ionic liquid [bmim] $[\mathrm{PF} 4]$ as polar microenvironment. Colloid Polym Sci. 2005;283(12):1371-5.

18. Li J, Zhang J, Han B, Zhao Y, Yang G. Formation of multiple water-in-ionic liquid-in-water emulsions. J Colloid Interface Sci. 2012;368(1):395-9.

19. Dobler D, Schmidts T, Klingenhofer I, Runkel F. Ionic liquids as ingredients in topical drug delivery systems. Int J Pharm. 2013;441(1-2):620-7. Epub 2012/11/06.

20. OECD Guideline for Testing of Chemicals. OECD Test Guideline 439: in vitro skin irritation-reconstructed human epidermis test method. OECD Publishing, Organization for Economic Cooperation and Development 2013.

21. OECD Guideline for Testing of Chemicals. OECD Test Guideline 431: in vitro skin corrosion: reconstructed human epidermis (Rhe) test method. OECD Publishing, Organization for Economic Cooperation and Development 2013.

22. OECD Guideline for Testing of Chemicals. Guidance document for the conduct of skin absorption studies. OECD Publishing, Organization for Economic Co-operation and Development 2004.

23. Diembeck W, Beck H, Benech-Kieffer F, Courtellemont P, Dupuis $\mathrm{J}$, Lovell $\mathrm{W}$, et al. Test guidelines for in vitro assessment of dermal absorption and percutaneous penetration of cosmetic ingredients. European Cosmetic, Toiletry and Perfumery Association. Food Chem Toxicol. 1999;37(2-3):191-205. Epub 1999/05/05.

24. Schlupp P, Weber M, Schmidts T, Geiger K, Runkel F. Development and validation of an alternative disturbed skin model by mechanical abrasion to study drug penetration. Results Pharma Sci. 2014:4:26-33. Epub 2014/01/01.

25. Cornellas A, Perez L, Comelles F, Ribosa I, Manresa A, Garcia MT. Self-aggregation and antimicrobial activity of imidazolium and pyridinium based ionic liquids in aqueous solution. J Colloid Interface Sci. 2011;355(1):164-71. Epub 2010/12/28. 
26. Barry BW, Saunders GM. Rheology of systems containing cetomacrogol 1000-cetostearyl alcohol. I. Self-bodying action. J Colloid Interface Sci. 1972;38(3):616-25.

27. Fatemi MH, Izadiyan P. Cytotoxicity estimation of ionic liquids based on their effective structural features. Chemosphere. 2011;84(5):553-63.

28. Stolte S, Arning J, Bottin-Weber U, Matzke M, Stock F, Thiele K, et al. Anion effects on the cytotoxicity of ionic liquids. Green Chem. 2006;8(7):621-9.

29. Weaver KD, Kim HJ, Sun JZ, MacFarlane DR, Elliott GD. Cytotoxicity and biocompatibility of a family of choline phosphate ionic liquids designed for pharmaceutical applications. Green Chem. 2010;12(3):507-13.

30. Azevedo AM, Ribeiro DM, Pinto PC, Lucio M, Reis S, Saraiva ML. Imidazolium ionic liquids as solvents of pharmaceuticals: influence on HSA binding and partition coefficient of nimesulide. Int J Pharm. 2013;443(1-2):273-8.

31. Vrikkis RM, Fraser KJ, Fujita K, Macfarlane DR, Elliott GD. Biocompatible ionic liquids: a new approach for stabilizing proteins in liquid formulation. J Biomech Eng. 2009;131(7):074514. Epub 2009/07/31.

32. Barry BW, Eccleston GM. Influence of gel networks in controlling consistency of $\mathrm{O} / \mathrm{W}$ emulsions stabilised by mixed emulsifiers. J Texture Stud. 1973;4(1):53-81.

33. Chen JS, Dickinson E. Effect of surface character of filler particles on rheology of heat-set whey protein emulsion gels. Colloids Surf B. 1999;12(3-6):373-81.

34. Chanamai R, Horn G, McClements DJ. Influence of oil polarity on droplet growth in oil-in-water emulsions stabilized by a weakly adsorbing biopolymer or a nonionic surfactant. J Colloid Interface Sci. 2002;247(1):167-76.
35. Philippova OE, Hourdet D, Audebert R, Khokhlov AR. Interaction of hydrophobically modified poly(acrylic acid) hydrogels with ionic surfactants. Macromolecules. 1996;29(8):2822-30.

36. Goddard ED, Ananthapadmanabhan KP. Interactions of surfactants with polymers and proteins. Boca Raton: CRC Press; 1993. 427 p. p.

37. Seddon KR, Stark A, Torres MJ. Influence of chloride, water, and organic solvents on the physical properties of ionic liquids. Pure Appl Chem. 2000;72(12):2275-87.

38. Sekar S, Surianarayanan M, Ranganathan V, MacFarlane DR, Mandal AB. Choline-based ionic liquids-enhanced biodegradation of azo dyes. Environ Sci Technol. 2012;46(9):4902-8.

39. Aalto TR, Firman MC, Rigler NE. p-Hydroxybenzoic acid esters as preservatives. I. Uses, antibacterial and antifungal studies, properties and determination. J Am Pharm Assoc (Wash). 1953;42(8):449-57.

40. Lück E, Jager M. Antimicrobial food additives: characteristics uses - effects. 2rd ed. Springer-Verlag: Berlin Heidelberg; 1997. p. $152-167$.

41. Lundov MD, Johansen JD, Zachariae C, Moesby L. Low-level efficacy of cosmetic preservatives. Int J Cosmet Sci. 2011;33(2):190-6.

42. Mizuuchi H, Jaitely V, Murdan S, Florence AT. Room temperature ionic liquids and their mixtures: potential pharmaceutical solvents. Eur J Pharm Sci. 2008;33(4-5):326-31. Epub 2008/02/23.

43. Holbrey JD, Reichert WM, Nieuwenhuyzen M, Sheppard O, Hardacre C, Rogers RD. Liquid clathrate formation in ionic liquid-aromatic mixtures. Chem Commun (Camb). 2003;(4):4767

44. Williams AC, Barry BW. Penetration enhancers. Adv Drug Deliv Rev. 2004;56(5):603-18. 